\title{
Avaliação da camada de fibras nervosas da retina nas afecções neuroftalmológicas da via óptica anterior
}

\author{
Retinal nerve fiber evaluation in neuro-ophthalmic \\ diseases of the anterior visual pathway
}

Mário Luiz Ribeiro Monteiro*

\begin{abstract}
Resumo
A avaliação da camada de fibras nervosas da retina tem grande importância no diagnóstico e acompanhamento de várias afecções da via óptica anterior. Nesta revisão, discutiremos os principais métodos de análise clínica e instrumental da camada de fibras nervosas da retina e revisamos os principais achados encontrados nas afecções da via óptica anterior incluindo lesões inflamatórias, isquêmicas, tóxicas, hereditárias, compressivas e traumáticas do nervo óptico, as lesões do quiasma óptico, as do trato óptico e aquelas do corpo geniculado lateral.

Descritores: Camada de fibras nervosas da retina; Neuropatias ópticas; Quiasma; Trato óptico; Corpo geniculado lateral; Tomografia de coerência óptica
\end{abstract}

\section{Abstract}

Retinal nerve fiber evaluation is important in the diagnosis and management of several diseases of the anterior visual pathway. In this report we review the clinical findings and the current techonologies avalilable to analyse the retinal nerve fiber layer. We furthermore review the main findings in several disease of the anterior visual pathways including inflammatory, ischemic, toxics, hereditary, compressive and traumatic optic neuropathies as well as lesion of the optic chiasm, optic tract and lateral geniculate body.

Keywords: Retinal nerve fiber layer; Optic neuropathies; Optic chiasm; Optic tract; Lateral geniculate body; Optical coherence tomography

* Universidade de São Paulo (USP) - São Paulo (SP), Brasil.

O autor declara inexistir conflitos de interesse

Recebido para publicação em 14/2/2011 - Aceito para publicação em 7/8/2011 


\section{INTRODUÇÃO}

A $\mathrm{s}$ afecções da via óptica anterior representam importantes diagnósticos em neuroftalmologia e incluem doenças de origem inflamatória, desmielinizante, isquêmica, compressiva, tóxicas, carenciais, hereditárias ou traumáticas que acometem os nervos ópticos, quiasma óptico, trato óptico e corpo geniculado lateral. A avaliação clínica destas afecções envolve tanto testes subjetivos da função visual, como a acuidade e o campo visual, quanto métodos objetivos que avaliem suas alterações estruturais.

A mensuração das alterações estruturais é de fundamental importância e pode ser realizada através do exame fundoscópico que permite uma estimativa grosseira da perda neural baseada no grau de palidez do disco óptico (DO) ou pela observação da camada de fibras nervosas da retina (CFNR). ${ }^{(1)}$ Após a observação inicial de Hoyt, vários estudos demonstraram que existe uma correlação entre o grau de anormalidade na função visual e a presença de defeitos na CFNR, mesmo em casos onde a palidez do disco óptico não é evidente..$^{(1-3)}$ Outros estudos, especialmente em pacientes com glaucoma, demonstraram também que o defeito na CFNR pode ser um sinal precoce de dano neural, podendo preceder o defeito de campo visual (CV). ${ }^{(4)}$ Portanto, a avaliação da CFNR tornou-se de método semiológico de fundamental importância em diversas afecções da via óptica anterior. ${ }^{(5-11)}$

A análise da CFNR é um elemento semiológico importante porque as alterações nestas fibras são mais fáceis de observar e estimar do que a palidez do DO especialmente em lesões discretas. Além disso, em alguns indivíduos o DO apresenta coloração naturalmente mais clara e é importante a observação da CFNR no sentido de valorizar ou não uma eventual "palidez" do disco.É importante também porque pode auxiliar no diagnóstico de diversas lesões da via óptica seja pela detecção da perda neural, seja pela identificação de padrões específicos de perda da CFNR. Por fim tal avaliação tem também importância na estimativa da possibilidade ou não de recuperação visual e no monitoramento do tratamento de algumas doenças.

Embora muita ênfase tenha sido dada nos últimos anos à avaliação da CFNR em pacientes com glaucoma, menor atenção foi destinada à importância deste dado semiológico em neuroftalmologia. Nesta revisão, após uma breve descrição da distribuição anatômica da CFNR e dos principais métodos semiológicos disponíveis para avaliação da CFNR, discutiremos as alterações encontradas nas principais afecções da via óptica desde o nervo óptico (excluído glaucoma) até o corpo geniculado lateral.

\section{Anatomia da camada de fibras nervosas da retina}

A CFNR é composta principalmente pelos axônios oriundos das células ganglionares da retina (CGR), juntamente com astrócitos e componentes das células de
Muller $^{12}$. A sua espessura é menor na periferia e maior ao redor do DO, principalmente nas margens superior e inferior, onde é em média de 200 micra. Já nos setores nasal e temporal ao redor do disco, a sua espessura é muito menor.A CFNR diminui também com a distância em relação ao disco óptico, se mostra inversamente proporcional à idade e parece não ter relação com a área do disco. ${ }^{(13)}$

Conhecer a distribuição anatômica dos axônios originados nas CGR e visibilizados nas porções mais internas da retina é de fundamental importância. Os axônios originados a partir das CGR na mácula nasal projetam diretamente em direção ao DO compondo parte do feixe papilomacular. Os axônios originados na região macular do lado temporal apresentam um padrão levemente arqueado em torno dos axônios maculares nasais, compondo as fibras remanescentes do feixe papilomacular. Os axônios das CGR nãomaculares que são nasais a fóvea tem um curso em linha reta ou levemente curvo em direção ao DO, enquanto que os axônios das CGR localizados temporalmente a fóvea apresentam um trajeto arqueado ao redor do feixe papilomacular e entram nos polos superior e inferior do DO. Portanto, a divisão da CFNR em partes temporal e nasal, é feita por uma linha vertical que passa através do centro da fóvea, e não no DO. As fibras que se originam das células ganglionares localizadas nasalmente à fóvea cruzam no quiasma óptico enquanto que as demais não cruzam. As fibras que não cruzam no quiasma óptico penetram o DO apenas nos polos superior e inferior, enquanto que as fibras originárias da retina nasal e que cruzam no quiasma penetram o disco óptico em toda sua volta (Figura 1). ${ }^{(12,14)}$ Ao nível do nervo óptico as fibras se agrupam na rima neuroretiniana, antes de iniciar o seu trajeto em direção às partes posteriores da via óptica. Geralmente é maior inferiormente, seguido pela região superior, depois nasal, e é mais fina temporalmente. ${ }^{(15)}$

Há também um arranjo específico das fibras em relação à sua disposição vertical visto em corte da retina. As fibras que se originam na retina periférica tendem a permanecer mais profundamente na retina enquanto que aquelas mais centrais assumem um trajeto mais superficial. Portanto, as fibras mais longas se situam em uma posição mais periférica enquanto que as mais curtas são mais superficiais na retina.

\section{Semiologia da camada de fibras nervosas retinianas}

$\mathrm{O}$ feixe de fibras nervosas é visível à oftalmoscopia como reflexos de fibras estriadas, mais facilmente visibilizadas com a pupila dilatada e usando uma luz aneritra $(540 \mathrm{~nm})$. A análise qualitativa da CFNR pode ser obtida além da oftalmoscopia também através do registro fotográfico (retinografia colorida ou com uso de filtros, luz anerita) ${ }^{(1)}$. Entretanto, estes métodos permitem apenas uma análise qualitativa, sendo dependentes da experiência do examinador e, portanto, altamente subjetivos. Além disso, Quigley et al. ${ }^{(16)}$ cha- 




Figura 1: Representação esquemática da camada de fibras nervosas da retina originárias da hemirretina temporal (em azul) e aquelas da hemirretina nasal (em vermelho)

maram a atenção para o fato de que, em alguns casos, até $50 \%$ das fibras nervosas podem ser perdidas sem que haja defeito na CFNR detectável através de retinografias. Em decorrência disso, a necessidade de métodos diagnósticos que permitissem uma quantificação objetiva da espessura da CFNR se fez necessário. Os principais instrumentos utilizados para avaliação da CFNR são: o polarímetro de varredura a laser, o laser confocal de varredura e a tomografia de coerência óptica.

\section{Polarímetro de varredura a laser}

$\mathrm{O}$ polarímetro com laser de varredura (GDx Laser Diagnostic Technologies, San Diego, CA, Estados Unidos) foi um dos primeiros aparelhos com a proposta de quantificar a CFNR peripapilar. A medida da espessura da CNFR baseia-se nas propriedades birrefringentes da CFNR. ${ }^{(17)} \mathrm{A}$ luz polarizada ao incidir sobre um meio birrefringente, sofre um retardo que é linearmente relacionado a espessura e propriedades ópticas do meio birrefringente.

A birrefringência da CFNR ocorre devido a disposição paralela dos microtúbulos e neurofilamentos axonais. ${ }^{(18)} \mathrm{O}$ GDx é constituído por um oftalmoscópio com laser de varredura, um modulador de polarização, um compensador de córnea e uma unidade de detecção de polarização. Sua capacidade de fornecer medidas quantitativas e reprodutíveis da camada da CFNR foi demonstrada em estudos experimentais e clínicos. ${ }^{(17,19,20)}$ Embora a maioria dos estudos clínicos utilizando o GDx tenham sido realizados em pacientes com glaucoma, muitas outras neuropatias ópticas foram estudadas, incluindo neurite óptica desmielinizante, ${ }^{(21)}$ trauma indireto do nervo óptico, ${ }^{(22)}$ e neuropatia óptica isquêmica, ${ }^{(23)}$ demonstrando que o GDx pode ser uma ferramenta útil na detecção da perda axonal em diversas afecções da via óptica anterior.

Um dos problemas com esta tecnologia é que qualquer estrutura birrefringente por onde passe o feixe de laser altera o retardo do feixe. No olho, a córnea e o cristalino têm birrefringência que pode ser variável de indivíduo para indivíduo. Em decorrência disso o aparelho incorpora um compensador da birrefringência corneana para evitar erros de interpretação. As primeiras versões dispunham de um compensador fixo de córnea (GDx-FCC) e a versão atual dispõe de um compensador variável da córnea (GDx-VCC) que estima e corrige o retardo devido a estruturas do segmento anterior do olho de tal forma e medir de forma mais acurada a CFNR peripapilar.

Estudos prévios demonstraram boa correlação entre defeitos do campo visual e medidas do GDx obtidas nas áreas superior e inferior peripapilar, ${ }^{(24)}$ no entanto, a capacidade deste instrumento para avaliar a espessura da CFNR nas regiões nasal e temporal foi questionada por alguns autores. ${ }^{(25,26)} \mathrm{Em}$ estudos utilizando pacientes com lesões quiasmáticas, demonstramos que o GDx falha na detecção da perda da CFNR no setor temporal do DO tanto na sua versão original ${ }^{(8)}$ quanto nas versões mais modernas com compensador variável de córnea (GDx-VCC) ou compensação ampliada da córnea (GDx-ECC). ${ }^{(27)}$ Desta forma, embora o aparelho possa ser útil na quantificação da CFNR média do DO, deve ser usado com cuidado quando o defeito de fibras for predominante no setor temporal do DO como ocorre com várias afecções neuroftalmológicas.

\section{Laser confocal de varredura (LCV)}

O LCV é um aparelho que utiliza a tecnologia do laser confocal, na qual um pequeno feixe de laser rapidamente escaneia a superfície retiniana horizontal e verticalmente. A luz refletida de cada ponto iluminado passa através de uma pequena abertura que é posicionada de tal forma que o plano focal da retina e aquele do sensor de luz estão opticamente conjugados. Uma série de imagens confocais é coletada e processada para calcular uma representação tridimensional da superfície da retina e do disco óptico.

O aparelho mais conhecido que utiliza o princípio do LCV é o tomógrafo retiniano de Heidelberg (HRT). O instrumento original (HRT I) foi seguido de modificações para o HRT II que usa um laser diodo de $670 \mathrm{~nm}$ e produz imagens de 384 X 384 pixels espalhados por uma área de 15 x 15 graus. Desta forma é possível analisar o tecido em camadas (ou secções), que podem variar de 16 a 64, dependendo da profundidade da estrutura tridimensional final.

O HRT fornece, portanto, imagens tridimensionais topográficas do DO e da CFNR adjacente ao disco. O software automaticamente calcula uma série de parâmetros incluindo a área do disco, área, profundidade e volume da escavação, área e volume da rima neural e a relação escavação disco. Estes cálculos são baseados em um plano de referência arbitrário situado $50 \mu \mathrm{m}$ abaixo da superfície do feixe papilomacular e também baseados em um contorno do disco determinado pelo exami- 
nador. Os resultados são altamente reprodutíveis e considerados precisos para medir alterações topográficas. Para estimar a CFNR o software do HRT mede a altura da retina ao longo de um contorno em volta do disco e subtrai a altura do plano de referência. Assim o HRT mede a superfície retina diretamente e apenas estima a CFNR indiretamente.

\section{Tomografia de coerência óptica}

A tomografia de coerência óptica (TCO) é uma modalidade de aquisição de imagem não-invasiva, que obtém imagens tomográficas de alta resolução, através de cortes transversais das estrutras internas da retina, por meio da medida de atraso do "eco luminoso" e a intensidade de reflexão da luz (próxima ao infravermelho - 820nm) nos tecidos. ${ }^{(28)}$ A construção da imagem é análoga a ultrassonografia modo B, só que usa a luz ao invés do som, baseia-se no princípio da inteferometria de baixa coerência. A resolução das imagens pode variar de acordo com a versão e tipo de aparelho, sendo na ordem de 10 micra para sua versão mais amplamente utilizada (Stratus-OCT; Carl Zeiss Meditec), mas podendo chegar na ordem de 5 micra nos aparelhos de nova geração, as TCO de alta resolução.

Atualmente duas variantes da tecnologia são disponíveis. A primeira utiliza a tecnologia domínio do tempo (TD, do inglês time-domain), disponível na versão comercial Stratus-OCT ${ }^{\mathrm{TM}}$ (Carl Zeiss Meditec Inc. Dublin, CA, EUA) que consegue resolução axial que varia de 128 a 768 pontos (A-scans), com um tempo de aquisição de 0,32 a 1,92 segundos respectivamente, dependendo da estratégia de aquisição utilizada. Tal tecnologia proporciona uma resolução axial menor do que $10 \mu \mathrm{m}$ e uma resolução transversal de $20 \mu \mathrm{m}$ aproximadamente. ${ }^{(29)}$

A habilidade da TCO-TD em produzir medidas quantitativas e reprodutíveis da CFNR tanto em olhos normais como doentes foi demonstrada previamente em diversos estudos clínicos. ${ }^{(30,31)}$ O protocolo de escaneamento para avaliação da CFNR mais amplamente utilizado é o fast RNFL thickness. Este protocolo consiste em três scans consecutivos $360^{\circ}$ ao redor do disco óptico com diâmetro de $3.4 \mathrm{~mm}$, cada scan é composto por $256 \mathrm{~A}$ scans e obtidos em um única sessão com 1.92 segundos de duração. Os valores obtidos são da espessura média da CFNR, em quatro quadrantes ( $90^{\circ}$ cada) e doze segmentos de $30^{\circ}$ cada. A espessura da CFNR é determinada pelo software do aparelho de acordo com a extensão da camada vermelha de alta refletividade que aparece junto a interface vitreorretiniana.${ }^{(93)}$ Existe no banco de dados do próprio aparelho uma escala normativa em cores para comparação em percentis, sendo verde para valores normais $(5<\mathrm{p}<95 \%)$, amarelo limítrofes $(1<\mathrm{p}<5 \%)$, vermelho diminuído $(0<\mathrm{p}<1 \%)$ e o branco (acima da média, $95<\mathrm{p}<100 \%$ ). O protocolo de análise disponível no próprio software do Stratus-OCT mais utilizado é $R N F L$ Thickness Avarage Analysis Report.

Aprimoramentos na tecnologia da TCO foram introduzidas recentemente, incluindo aparelhos de alta resolução, que utilizam o princípio do domínio de Fourier (FD do inglês Fourier Domain) para geração de imagens a uma velocidade de varredura mais alta. Imagens seccionais da retina com uma resolução até 5 vezes maior que a TCO convencional podem assim ser adquiridas. Esse aumento da resolução e da velocidade de varredura permite uma captura de imagens da alta densidade dos tecidos retinianos enquanto minimiza os artefatos gerados pela movimentação ocular. A TCO-FD ao invés de ajustar a posição do espelho de referência, grava a informação interferométrica usando um espectrômetro, gerando uma velocidade de varredura de 18.000 até 25.000 scans/ segundo do tipo A, com resolução axial de $5 \mu \mathrm{m}$ e uma resolução horizontal de $20 \mu \mathrm{m}$ ou menor. Essa tecnologia fornece também varreduras em scan B em alta resolução (até 4096 pixels de resolução) e imagens volumétricas tridimensionais que cobrem uma área da retina de até 6 x $6 \mathrm{~mm}$. O aumento da resolução axial permite melhor visualização das camadas intrarretinianas como a dos fotorreceptores, das plexiformes, nucleares, das células ganglionares e da CFNR. ${ }^{(32-36)}$

\section{Tipos de alterações da camada de fibras nervosas}

Quando um axônio é lesado de forma irreversível, ocorrem dois tipos de degeneração: a anterógrada (walleriana) e a retrógada. ${ }^{(37)}$ A degeneração anterógrada ocorre na porção distal do axônio que foi separado do seu corpo celular, enquanto que a degeneração retrógada ocorre no segmento proximal do nervo que permanece em contato com corpo celular. Apesar da degeneração anterógrada se tornar próximo de completa dentro de 7 dias após a lesão, a porção do axônio que permanece conectada ao corpo celular, e o próprio corpo celular, mantém a aparência normal por 3 a 4 semanas. Após este período, entretanto, a estrutura restante inteira (o corpo celular e axônio a partir do ponto da lesão) degenera rapidamente, tanto que por volta de $6 \mathrm{a}$ 8 semanas após uma lesão grave ao nervo óptico, nenhuma célula ganglionar afetada permanece viável. ${ }^{(37,38)}$ Com resultado deste processo degenerativo conseqüente a lesão da fibra nervosa e morte da CGR, aparecem os defeitos na CFNR e palidez do DO.

Como já mencionado a palidez é difícil de ser quantificada, sendo assim, a perda axonal é mais facilmente estimada observando-se a CFNR peripapilar.Tal visibilização, no entanto, exige treinamento clínico e observação cuidadosa sendo este o motivo pelo qual o assunto passa despercebido por grande parte dos oftalmologistas. Dois padrões principais de perda axonal ocorrem: a perda difusa e a perda localizada.

\section{Perda difusa da camada de fibras nervosas}

A perda completa da CFNR é facilmente reconhecida pela exposição de detalhes anatômicos da retina e da coróide. Assim pequenos vasos e detalhes da retina que normalmente são obscurecidos pela CFNR se tornam aparentes. A retina sem fibras assume um padrão mosqueado. As margens do disco óptico se tornam 


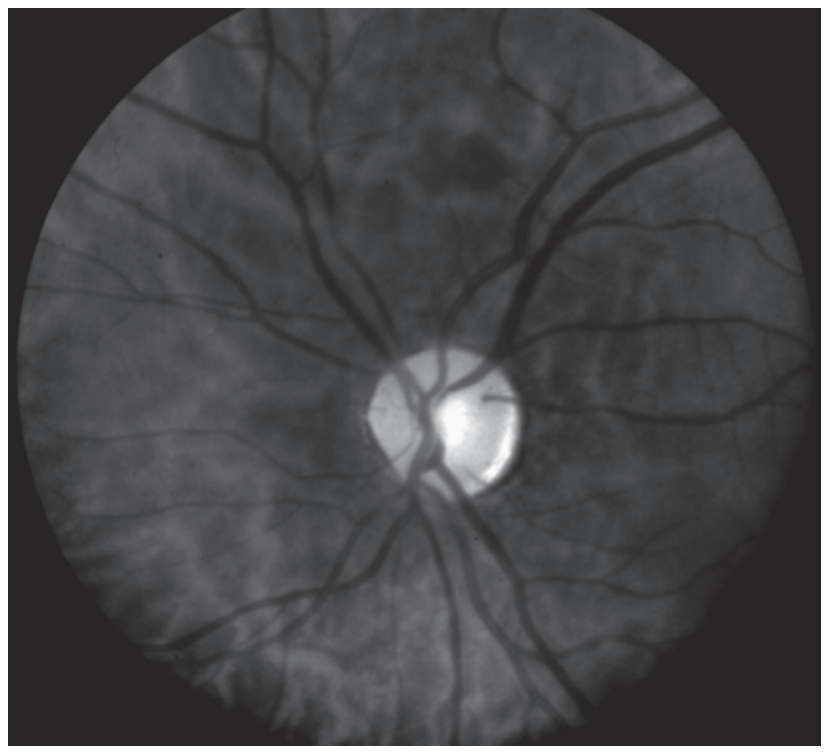

Figura 2: Perda difusa da camada de fibras nervosas retiniana: falta de estriações peripapilares e exposição dos vasos retinianos

muito bem definidas (Figura 2).

Perda difusa e parcial das fibras que seja uniformemente distribuída pelo nervo é muito mais difícil de perceber ao exame clínico, principalmente se for bilateral. Casos parciais unilaterais são melhor avaliados do ponto de vista clínico pela comparação com o olho contralateral seja à oftalmoscopia, seja através de fotografias.

\section{Perda focal das fibras nervosas retinianas}

Perda focal significa redução das fibras em uma região circunscrita da via óptica anterior e sua parte correspondente na CFNR. A aparência do defeito depende da profundidade, largura e posição em relação ao disco óptico. ${ }^{(2)}$ Defeitos profundos e de toda espessura são mais fáceis de diagnosticar que os parciais. Os defeitos focais são delimitados por curvas que acompanham o arranjo curvilinear da CFNR nos feixes de fibras e sempre reduzem em direção ao DO (Figura 3).

Defeitos focais pequenos são geralmente melhor vistos a uma distância 1 a 3 discos ópticos afastada da cabeça do nervo óptico, sendo mais reduzidos próximo ao disco óptico. Defeitos mais largos, em forma de cunha, podem ser vistos até uma distância maior.

A distribuição dos defeitos focais da CFNR depende do local da lesão na via óptica anterior. Um acometimento frequente é aquele que envolve o feixe papilo-macular, com perda importante das fibras no setor temporal do disco óptico (Figura 4).

Do ponto de vista prático, o tipo de defeito pode ser dividido nas lesões do nervo óptico, do quiasma óptico, do trato óptico e do corpo geniculado lateral.

\section{Alterações na camada de fibras nervosas nas lesões dos nervos ópticos}

Defeitos focais são frequentemente encontrados em

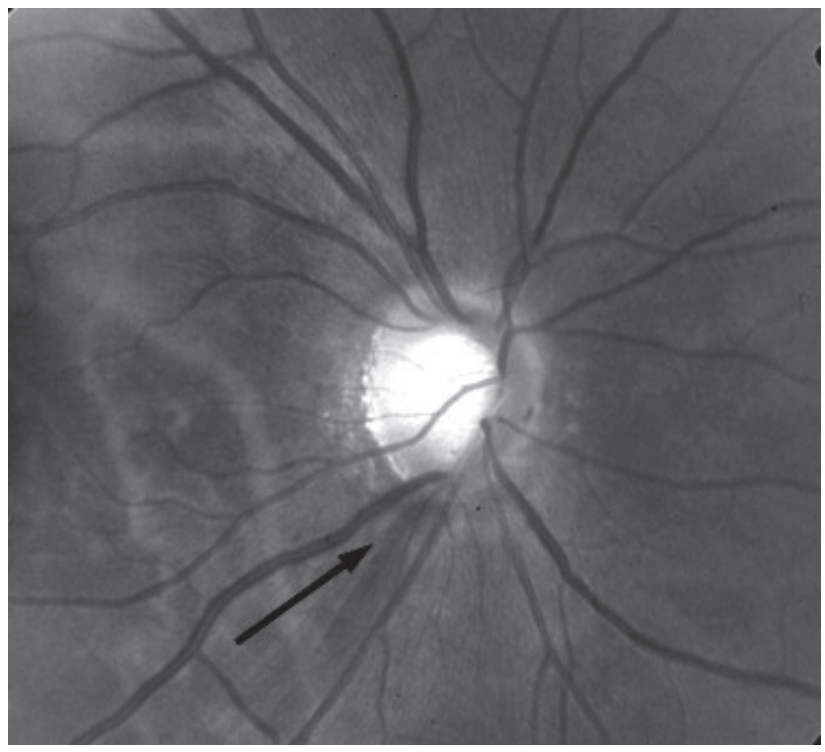

Figura 3: Representação de um defeito focal na camada de fibras nervosas da retina (seta)

vários tipos de lesões do nervo óptico, embora a maioria das lesões possa também causar atrofia difusa das fibras, principalmente nas fases mais avançadas da doença. $\mathrm{O}$ tipo de perda de fibra representa assim a morte axonal decorrente de qualquer tipo de lesão. As principais neuropatias ópticas são representadas pelas neuropatias inflamatórias/ desmielinizantes, isquêmicas, hereditárias, tóxicas, carenciais, compressivas e traumáticas.

\section{Neuropatias inflamatórias}

As neuropatias inflamatórias são frequentes e englobam as doenças desmielinizantes e imuno-mediadas, idiopáticas ou infecciosas. Na fase aguda o DO pode ser normal ou apresentar edema que geralmente é discreto a moderado. Exsudatos podem ocorrer e hemorragias são incomuns. A perda da CFNR começa após um mês da crise de neurite óptica e continua por 3 a 6 meses quando então aparecem os defeitos na camada de fibras. Os defeitos podem ser focais ou difusos, ocorrendo em até $80 \%$ dos pacientes com neurite óptica. ${ }^{(10)}$ Existe uma preferência para o acometimento do setor temporal, com perda importante do feixe papilo-macular (Figura 4).

A TCO é um método importante na quantificação da lesão axonal decorrente de neurite óptica aguda e já foi bastante investigado em casos onde o acometimento do nervo óptico está associado à esclerose múltipla (EM). Quando comparado a controles normais os olhos acometidos por neurite óptica apresentam uma redução de $33-46 \%$ da CFNR à TCO ${ }^{(39,40)} \mathrm{O}$ afilamento é predominantemente no quadrante temporal, envolvendo o feixe papilomacular e se torna evidente de 3 a 6 meses após o episódio agudo. ${ }^{(40)}$ Pacientes com perda mais grave da CFNR e com afilamento intenso da mesma tem menor chance de recuperação visual. ${ }^{(11)}$ Costello et al. concluíram que uma medida de CFNR de $75 \mu \mathrm{m}$ ou menos implica num prognóstico ruim quanto à 


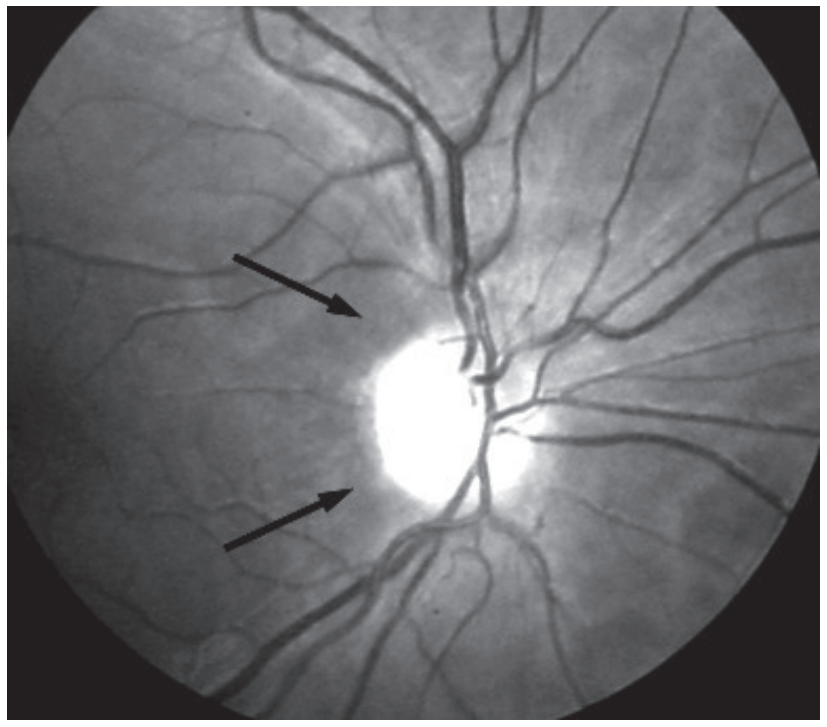

Figura 4: Perda da camada de fibras nervosas predominante no setor temporal do disco óptico. Observe a ausência de estriações referentes às fibras nervosas entre as setas

recuperação visual. ${ }^{(40)}$ Perdas menores mas significantes também já foram observadas em olhos contralaterais àqueles que tiveram neurite óptica em pacientes com esclerose múltipla indicando a existência de lesão subclínica naquela condição. ${ }^{(41)}$

Uma vez que a retina é o único lugar onde os axônios podem ser visibilizados diretamente, a quantificação da CFNR abriu um potencial muito grande de avaliação de neurodegeneração existente na EM, ou seja uma janela para a monitorização da doença. Vários estudos identificaram que em olhos de pacientes com EM que tiveram neurite óptica existe uma perda da CFNR em média de 20 a $38 \mu$ m, enquanto que em olhos assintomáticos dos mesmos pacientes existe uma perda em torno de $7 \mu \mathrm{m}$. Estes estudos enfatizam a importância de se examinar cuidadosamente a CFNR nos pacientes com EM, especialmente quando se tenta monitorar o efeito de tratamentos destinados a prevenir ou reduzir a perda neural nesta afecção. ${ }^{(42)}$ Embora a maior parte dos estudos tenha sido feita usando a TCO outros investigadores documentaram também a utilidade do polarímetro de varredura a laser na avaliação dos pacientes com esclerose múltipla. ${ }^{(21,43,44)}$

A neurite óptica pode também fazer parte da neuromielite óptica (NMO) uma condição idiopática, desmielinizante e necrotizante do sistema nervoso central que acomete o nervo óptico e a medula espinhal. Estudos recentes indicam que se trata de uma doença distinta da EM, caracterizada pela presença de anticorpos antiaquaporina 4. Embora tradicionalmente tenha sido considerada uma condição monofásica e bilateral, atualmente se sabe que o acometimento do nervo óptico pode ser uni ou bilateral e pode ocorrer antes ou depois da mielite transversa. Na fase aguda geralmente há edema do DO e na fase crônica existe palidez de papila que pode ser desde discreta até muito grave. $\mathrm{O}$ prognóstico visual é geralmente mais grave do que na neurite óptica da NMO do que naquela de causa idiopática ou associada a esclerose múltipla. ${ }^{(45)}$

Estudos usando TCO mostram que a CFNR se mostra significantemente reduzida em pacientes com NMO comparada a controles ou pacientes com esclerose múltipla. ${ }^{(46)} \mathrm{O}$ afilamento envolve os 4 quadrantes do disco, com predominância de acometimento dos quadrantes superior e inferior quando comparado à EM. Por outro lado, na EM o acometimento é preferencial no setor temporal do DO. ${ }^{(11,40)}$

Desta forma nas neurites ópticas a quantificação da perda neural é importante não apenas para o diagnóstico da perda neural existente na doença seja no olho acometido seja no olho contralateral de indivíduos com esclerose múltipla como também serve para monitorar evolução da doença principalmente quando se administram novos tratamentos.

\section{Neuropatias ópticas isquêmicas}

A neuropatia óptica isquêmica mais comumente afeta a porção anterior do nervo óptico e se caracteriza por edema de disco na fase aguda e palidez significativa algumas semanas depois, sem recuperação visual importante. Esta forma é denominada neuropatia óptica isquêmica anterior (NOIA) e pode ser classificada em de causa arterítica e não arterítica. (NOIA-NA). Enquanto a forma arterítica se mostra decorrente do envolvimento das artérias ciliares posteriores por arterite temporal, a fisiopatogenia da forma não arterítica é conhecida apenas em parte, acreditando-se ser uma doença multifatorial.

A NOIA-NA é a neuropatia óptica mais comum no idoso. Acredita-se que o local do enfarto do nervo óptico se situa na porção retrolaminar do nervo, suprida pelas artérias ciliares posteriores curtas. Várias hipóteses etiopatogênicas incluem a hipoperfusão da microcirculação ou defeito na autoregulação dos vasos. A afecção geralmente acomete discos pequenos, os chamados "disco de risco". ${ }^{(47)} \mathrm{Na}$ fase aguda ocorre edema do DO geralmente associado a hemorragias peripapilares. O edema pode ser difuso ou segmentar, neste caso mais comumente no pólo superior do disco óptico. Na fase aguda a TCO revela aumento da CFNR consistente com edema do DO e da retina peripapilar. ${ }^{(48)}$ Quatro a seis semanas após o evento o edema de papila regride e aparece a palidez do disco. A perda de CFNR pode ser segmentar superior (mais comum) (Figura 5) ou difusa. Ocorre afilamento dos vasos retinianos, redução da rima neural e perda da CFNR. O afilamento se desenvolve ao longo de alguns meses e estabiliza 6 meses após a crise. ${ }^{(49)} \mathrm{O}$ laser confocal de varredura também já foi usado para avaliar o nervo óptico na fase crônica da NOIA-NA e mostrou afilamento significativo da CFNR comparado com normal. ${ }^{50}$ Estes métodos usualmente mostram afilamento da CFNR não apenas no local onde existe atrofia óptica visível mas também 


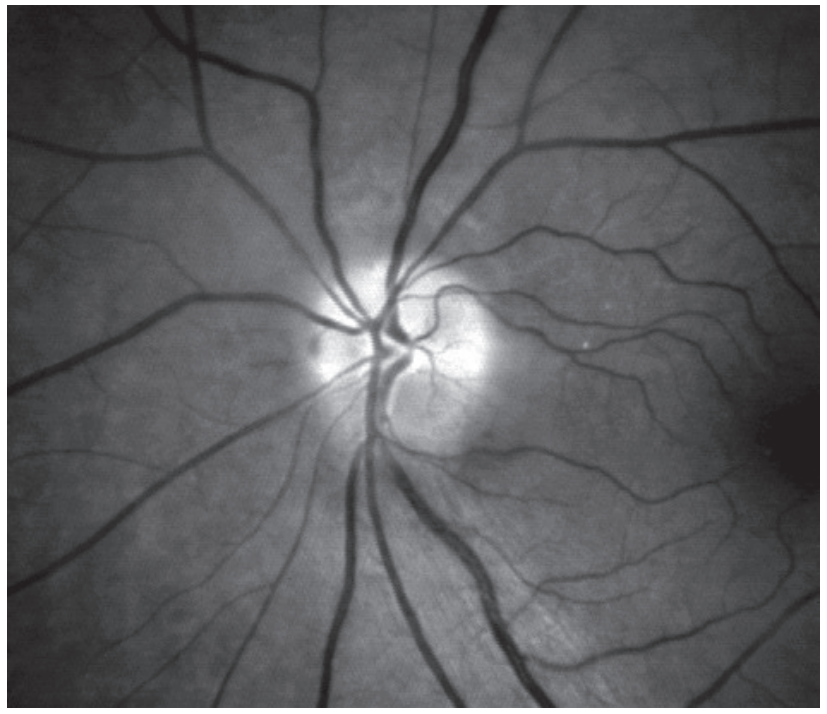

Figura 5: Palidez e perda da camada de fibras nervosas no setor superior do disco óptico após neuropatia óptica isquêmica; Observe estreitamento arteriolar na região superior

em outros setores do DO.

A análise quantitativa do DO revela na fase crônica afilamento significativo da CFNR, que se desenvolve ao longo de meses até estabilizar 6 meses após o evento. Estudos com o LCV indicam que olhos afetados tem redução importante da CFNR quando comparada com normais. ${ }^{50}$ Estudos com a TCO também mostram perda da CFNR e evidenciam que após a NOIA existe perda da CFNR não apenas no setor acometido pela perda de campo visual indicando que a perda de fibras pode ir além do local de palidez do DO ${ }^{(51)} \mathrm{O}$ afilamento da CFNR também pode ser bem demonstrado na fase crônica da doença através do GDx. ${ }^{(23,52)}$

\section{Neuropatias compressivas}

Compressões do nervo óptico por lesões na órbita, no canal óptico ou no nervo óptico intracraniano produzem perda de acuidade visual, visão de cores e defeito de campo visual. A perda visual é lenta e progressiva e pode haver proptose associada nos casos de lesões orbitárias.

As lesões compressivas podem ser causadas por tumores orbitários extrínsecos ao nervo óptico, tumores do nervo óptico como o meningioma e o glioma, orbitopatia distireoidiana e displasias ósseas. Em quase todos os casos a perda é muito lenta e quase nunca existe uma fase aguda da neuropatia. O aspecto do nervo óptico depende do estádio da apresentação, do local da compressão e da duração da compressão. Lesões infiltrativas devido a doenças hematológicas malignas como leucemias e linfomas também podem ocorrer.

Embora na fase aguda possa ocorrer edema do DO na maioria destas neuropatias, ${ }^{(53-56)}$ a sua característica principal é a do desenvolvimento progressivo de perda da CFNR que pode ser identificada e quantificada seja pela polarimetria com laser de varredura seja pela TCO.

\section{Neuropatias hereditárias}

As neuropatias ópticas hereditárias representam um grupo de afecções do nervo óptico diretamente relacionadas a herança genética. Podem ser isoladas, associadas a outros sinais neurológicos e sistêmicos e podem também fazer parte de uma doença neurológica degenerativa como as mucopolissacaridoses e as lipidoses. Do ponto de vista do oftalmologista as principais neuropatias hereditárias são: a atrofia óptica dominnante e a Doença de Leber.

A atrofia óptica dominante (AOD) é a neuropatia óptica hereditária mais comum. O quadro clínico se caracteriza por início insidioso, ocorrendo na maioria dos casos antes dos 10 anos de idade, embora usualmente os pacientes não sejam capazes de dizer com exatidão quando começou a afecção. Geralmente há piora lenta e progressiva da acuidade visual com a idade. A doença é bilateral, caracterizada por redução da acuidade visual, leve a moderada (em torno de 20/60), podendo variar de 20/ 20 a conta dedos. A perda visual geralmente é reduzida de forma semelhante nos dois olhos, sendo que a grande maioria permanece com acuidade visual melhor que 20/ 200. Uma grande proporção de casos pode ser atribuída a uma mutação no gene denominado OPA 1 e a incidência estimada é de 1:10.000 a 1:50.000 indivíduos.

O exame clínico característico inclui ainda a redução na visão de cores e a presença de escotomas centrais, paracentrais ou cecocentrais ao campo visual que geralmente são pequenos. Ao fundo de olho se observa palidez de papila que pode ser discreta no setor temporal ou ser mais difusa e acentuada. Pode ocasionalmente haver escavação da papila causando confusão com glaucoma. ${ }^{(57)} \mathrm{Na}$ AOD ocorre perda difusa da CFNR mas mais acentuada no setor temporal do disco óptico. Este achado é consistente com perda preferencial do feixe papilomacular. ${ }^{(57,58)}$ Geralmente não ocorrem defeitos em fenda ou cunha nas arcadas como costuma ocorrer no glaucoma ou mesmo na esclerose múltipla. Este padrão de perda de fibras já foi documentado de forma adequada pela TCO que pode ser útil para monitorar a progressão da doença e auxiliar na diferenciação com outras neuropatias, como o glaucoma, na qual o acometimento do setor temporal não ocorre ou é muito tardio. Kim e Hwang avaliaram 32 olhos de 16 pacientes com AO dominante e encontraram defeito no quadrante temporal do disco óptico em 90,6\% dos olhos achado estes bastante diferentes do que se observa no glaucoma. ${ }^{(58)}$

A neuropatia óptica hereditária de Leber (NOHL) é uma doença mitocondrial de transmissão materna que se caracterizada por perda visual de evolução aguda ou subaguda. A doença é bilateral e causa perda grave da visão ocorrendo geralmente em indivíduos do sexo masculino, com idade preferencial da segunda à quarta década de vida. A predominância pelo sexo masculino varia entre 80 a $90 \%$ na maioria dos pedigrees relatados.

A perda visual geralmente se inicia de forma indolor em um dos olhos. O segundo olho é afetado se- 
manas ou meses depois. Quase todos os pacientes terão o acometimento do segundo olho em um período de 1 ano. Geralmente a progressão da perda visual é subaguda, com deterioração da função visual ao longo de semanas ou meses até a sua estabilização. A acuidade visual varia desde ausência de percepção luminosa até 20/20, embora a maior parte dos pacientes tenha acuidade visual pior que 20/200. A visão de cores também é afetada, mas geralmente junto com a perda de acuidade visual. As reações pupilares mostram um defeito pupilar aferente, embora possam estar relativamente preservadas, quando comparado ao acometimento em outras neuropatias. Os defeitos de campo visual geralmente são do tipo escotoma central ou cecocentral. O escotoma pode ser relativo nas fases iniciais da doença mas logo se torna denso e absoluto.

$\mathrm{Na}$ maioria dos pacientes com NOHL a perda visual permanece grave e fica permanente. No entanto, alguns indivíduos podem apresentar recuperação visual, que pode ser importante, mesmo meses ou anos após a perda visual. A recuperação pode ser na forma de uma melhora muito gradual da visão central, às vezes restrita a uma pequena ilha central de recuperação da visão. A recuperação visual ocorre com maior freqüência em algumas do que em outras mutações mitocondriais.

Pelo menos quatro mutações já foram identificadas, nas posições de número 3.460, 11.778, 14484 e 15.257 do DNA mitocondrial, que é importante para a formação de diversas enzimas do sistema produtor de energia da célula, a fosforilação oxidativa. Três delas são denominadas mutações primárias e respondem por pelo menos $90 \%$ dos casos de NOHL, localizadas nas posições 11778 (69\% dos casos), 3460 (13\% dos casos) e 14484 (14\% dos casos). Não há nenhuma medida terapêutica efetiva conhecida para a NOHL mas pode ocorrer melhora espontânea principalmente nos portadores da mutação 14484 .

As alterações no DO e na CFNR podem ser divididas em fases aguda e crônica. Na fase aguda, o exame fundoscópico pode mostrar edema na CFNR, hiperemia do disco óptico com pseudo-edema de papila e vasos telangiectásicos na retina peripapilar. Este aspecto é bastante típico e se caracteriza pela presença de microangiopatia telangiectásica peripapilar associada à edema da camada de fibras nervosas, mas com ausência de extravasamento de contraste na angiofluoresceinografia. Comunicações arteriovenosas em graus variáveis podem ser vistas à angiofluoresceinografia. Tais achados estão presentes na fase sintomática na doença, nas primeiras semanas ou meses, tendendo a desaparecer em seguida. Alguns pacientes, no entanto, quando examinados na fase aguda tem o disco óptico e CFNR de aspecto normal, não apresentando os achados acima. ${ }^{(59,60)}$

Com o tempo as arteríolas e capilares do lado temporal do disco óptico se tornam estreitados e começa a haver a perda axonal no feixe papilomacular. O edema do $\mathrm{DO}$ desaparece e dá lugar à palidez do disco óptico e rarefação da CFNR, inicialmente acometendo o setor temporal depois progredindo para os demais setores. Na fase crônica, pode também ocorrer escavação do disco óptico o que também pode levar a confusão com neuropatia óptica glaucomatosa. No entanto, diferente do glaucoma no qual a rima neuroretiniana remanescente tem cor normal na NOHL existe palidez acentuada do setor temporal. Além disso, a profundidade e grau de escavação não é tão acentuada como no glaucoma.

A TCO mostra na fase aguda espessamento da CFNR correspondendo ao achado clínico de edema do disco. Na fase atrófica há afilamento acentuado da CFNR documentado pela TCO. Barboni et al. ${ }^{(61)}$ avaliaram a CFNR de 38 pacientes com NOHL, classificados em fase precoce, quando com menos que 6 meses de duração da doença ( 8 pacientes) fase atrófica nos demais. A CFNR foi avaliada com a TCO-TD e foi encontrado um espessamento da espessura média da CFNR assim como no quadrante superior, inferior e nasal nos olhos na fase aguda. Na fase crônica os olhos mostraram afilamento da CFNR em todas as regiões. Estes dados indicam que o feixe papilomacular parece acometido mais precocemente do que os demais setores do disco óptico. ${ }^{(61)}$

\section{Neuropatias tóxicas e carenciais}

As neuropatias ópticas tóxicas e carenciais se manifestam de forma semelhante no disco óptico, decorrente de dano de causa nutricional e/ou tóxica. ${ }^{(62)}$ De maneira geral, estas afecções apresentam em comum perda visual geralmente bilateral, indolor, insidiosa e progressiva, ou, em casos específicos, de aparecimento súbito (como no caso da ingestão de metanol, por exemplo), com perda de campo visual com características bem típicas como escotoma central ou cecocentral, preservando a periferia. Apesar de nos estágios iniciais a aparência do disco óptico ser normal ou levemente hiperemiado, nas fases mais tardias da doença é que encontramos as alterações oftalmoscópicas mais características, a palidez de disco. O grau de palidezé variável, mas existe uma preferência para a palidez ser mais proeminente no setor temporal do disco.

A maioria das lesões é bastante rara, sendo a mais importante a neuropatia óptica do alcoólatra, também chamada ambliopia tabaco-alcool, embora o efeito do fumo seja questionável e todas as evidências indiquem que se trate na verdade de uma neuropatia óptica carencial, bilateral e simétrica que parece ser devido a combinação dos efeitos do cianeto decorrente da exposição ao tabaco e a redução da vitamina B12 e folato secundário ao consumo de etanol. No entanto, a neuropatia óptica ocorre também em alcoólatras que não são fumantes. ${ }^{(63)}$

Na fase aguda o aspecto do DO é normal ou se observa discreto edema. Nas fases mais avançadas ocorre redução progressiva da CFNR, particularmente no feixe papilomacular (Figura 6). Quando o tratamento é instituído geralmente existe melhora visual a não ser nas fases avançadas da doença onde há atrofia grave do feixe papilomacular.

ATCO mostra afilamento da CFNR no setor temporal associada a aumento da espessura das fibras nos 

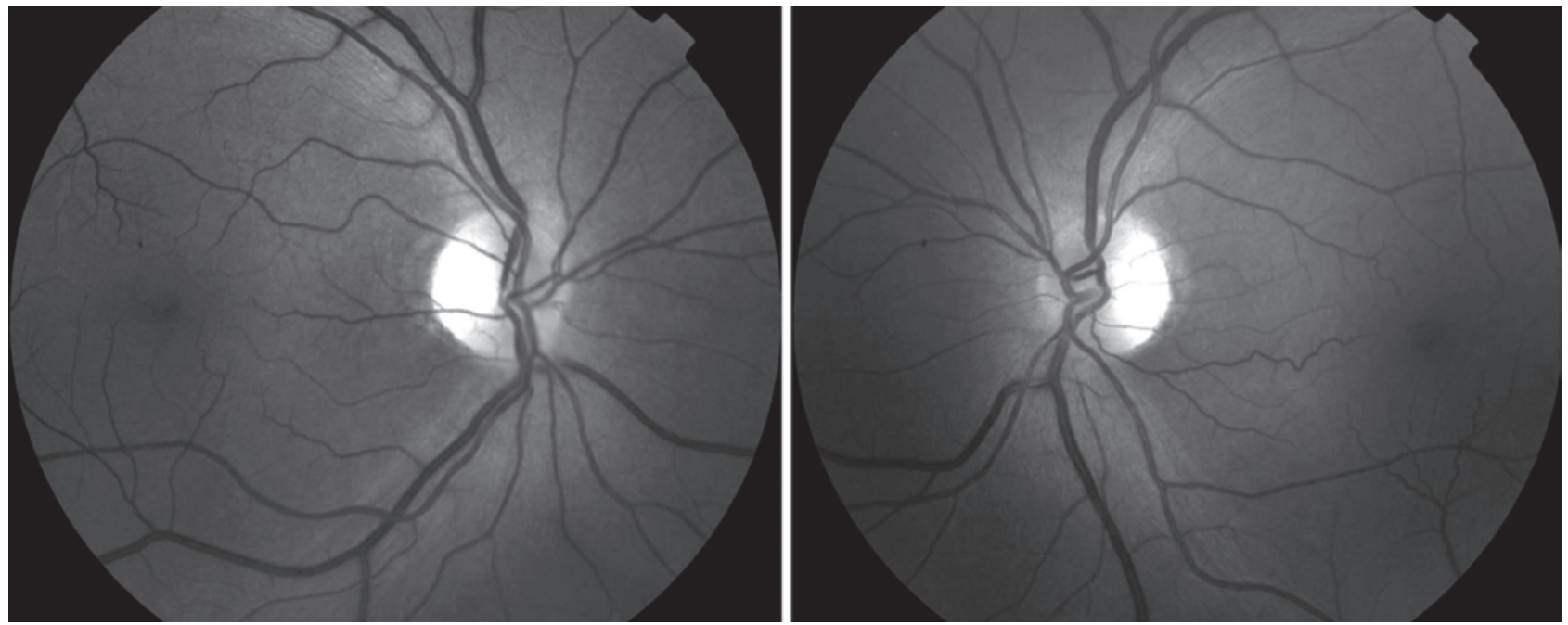

Figura 6: Fotografia de paciente com neuropatia óptica tabaco-alcool com palidez e perda da camada de fibras nervosas retinianas no setor temporal de ambos os olhos

demais quadrantes. ${ }^{(64)}$ Com o tempo a elevação da CFNR normaliza e geralmente persiste o afilamento temporal. ${ }^{(65)}$ Recentemente avaliamos 3 pacientes com perda visual progressiva associada a alcoolismo e carência nutricional através da TCO-TD. ${ }^{(66)}$ Dois pacientes demonstraram perda acentuada da CFNR no setor temporal do disco óptico em correspondência com o escotoma cecocentral que apresentavam (Figura 7).

No entanto, um terceiro paciente tinham medidas da CFNR dentro dos limites da normalidade ou até mesmo aumentada em alguns setores, com base nos dados normativos do aparelho, de forma semelhante ao que já foi observado em outras neuropatias tóxicas. ${ }^{(6)}$ Estes achados indicam que de forma análoga ao que se observa na fase aguda da NOHL e de algumas neuropatias tóxicas o aumento da CFNR nas fases iniciais pode dificultar a observação de eventual perda neural associada que só será percebida quando da redução do edema axonal na fase crônica. Este conhecimento é importante para evitar confusão diagnóstica.

\section{Neuropatia óptica traumática}

A neuropatia óptica traumática (NOT) é uma condição grave que pode ser uni ou bilateral e pode ocorrer por mecanismo direto ou indireto. Traumatismo direto resulta de lesões penetrantes na órbita enquanto que o trauma indireto resulta de traumatismo fechado no qual a onda de choque atua no canal óptico levando à perda visual. A perda visual geralmente ocorre associada a lesões frontais ou temporais. Pode haver melhora visual, particularmente no trauma indireto do nervo óptico, mas isso nem sempre ocorre.

Na fase aguda, no caso dos traumatismos posteriores na órbita ou ao nível do canal óptico, o DO tem aspecto normal. Algumas semanas depois ocorre atrofia progressiva das fibras nervosas o que pode ser demonstrado clinicamente e com o uso do polarímetro de varredura a laser ou pelo uso da TCO.$^{(67,68)} \mathrm{Em}$ um estudo recente observando sequencialmente 3 pacientes com trauma indireto do nervo óptico que foram avaliados semanalmente por 5 semanas e também ao redor da décima segunda semana após o trauma. Todos os pacientes apresentaram redução progressiva da CFNR evidenciada na segunda semana após o trauma e se acentuando na quarta semana mas se tornando mais acentuada 12 semanas depois. ${ }^{(68)}$ $\mathrm{Na}$ fase crônica, portanto, existe atrofia das fibras e do nervo óptico com perda irreversível da visão em diferentes graus dependendo da gravidade da lesão. Nos casos graves a palidez do disco óptico se torna aparente 3 a 4 semanas após o trauma e progride para atrofia completa. Em casos mais leves a palidez ocorre mais tarde e pode ser discreta. $\mathrm{Na}$ fase crônica a escavação do disco é incomum mas pode ocorrer. Observa-se perda difusa da CFNR que pode ser quantificada pela polarimetria de varredura a laser ou pela TCO. ${ }^{(68)}$

\section{Lesões do quiasma óptico}

Lesões compressivas do quiasma óptico são mais comumente decorrentes de tumores hipofisários. Outras causas incluem os craniofaringeomas, meningioma supraselar e aneurismas. A característica clínica mais importante é o desenvolvimento de hemianopsia bitemporal decorrente do acometimento preferencial das fibras cruzadas. ${ }^{(69)}$ As alterações no disco óptico estão ausentes no começo do quadro e se manifestam quando existe lesão axonal irreversível. A disposição das fibras nervosas da retina no quiasma óptico é responsável pelo padrão da perda axonal na compressão quiasmática. As fibras da retina temporal atravessam diretamente o quiasma óptico em direção ao trato óptico ipsilateral, enquanto as fibras da retina nasal cruzam o quiasma óptico para o trato óptico contralateral.

Lesões quiasmáticas extensas acometendo a porção mediana do quiasma e afetando as fibras cruzadas 
RNFL THICKNESS AVERAGE ANALYSIS

OD

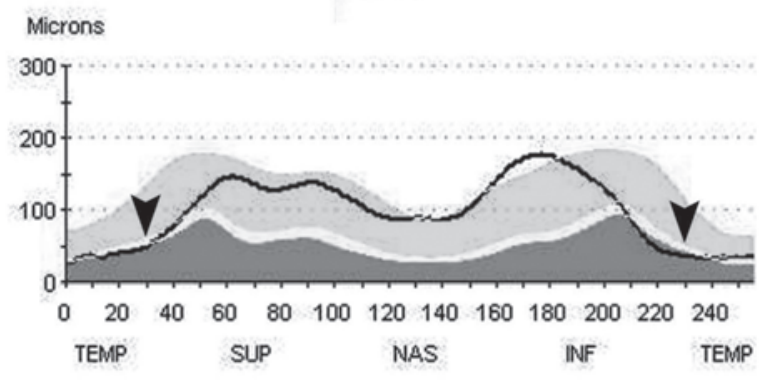

\section{OS}

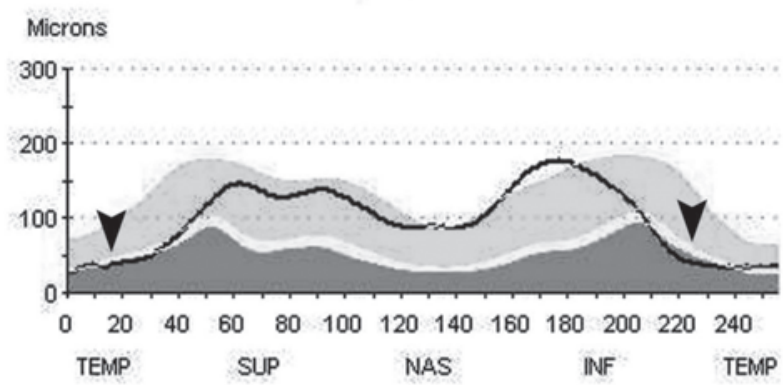

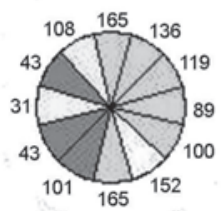
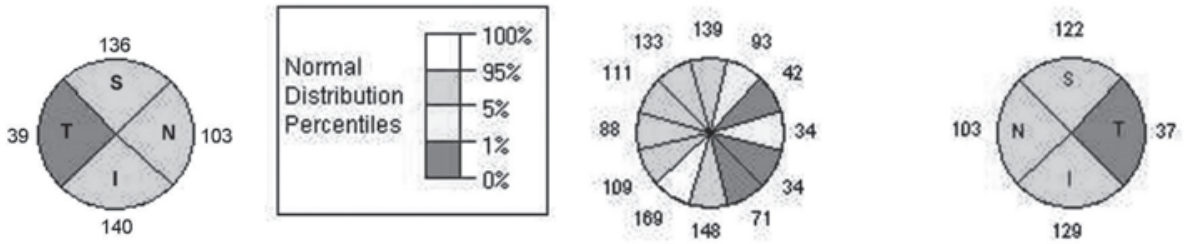

Figura 7: Tomografia de coerência óptica de paciente com neuropatia tabaco-álcool evidenciando perda das fibras no setor temporal de ambos os olhos (setas)

de forma grave com preservação das fibras não cruzadas geralmente apresentam sinais característicos ao fundo de olho. As fibras que cruzam no quiasma tem seus corpos celulares na retina nasal e são lesados enquanto que as fibras da hemirretina temporal, que penetram o disco óptico superior e inferiormente são preservadas. A representação oftalmoscópica da perda de fibras nervosas da hemirretina nasal é conhecida como atrofia em banda do nervo óptico, onde se observa palidez do disco óptico nos setores nasal (correspondendo às fibras da retina nasal periférica) e temporal (correspondendo às fibras maculares nasais) associada à rarefação do aspecto estriado da CFNR nos mesmos setores. ${ }^{(70)}$ Mikelberg e Yidegiligne relataram que, embora ocorra à preservação das fibras da retina temporal que penetram o disco óptico pelas arcadas superior e inferior, existe perda axonal significativa também nestas arcadas, uma vez que parte das fibras nervosas destes setores também se originam da retina nasal, cujas fibras são acometidas no quiasma óptico( ${ }^{71)}$ (Figura 8).

Clinicamente, a atrofia em banda do nervo óptico em pacientes com compressão quiasmática pode ser utilizada como parâmetro para o prognóstico visual. Assim, enquanto que os pacientes que apresentam atrofia do nervo óptico podem mostrar algum grau de recuperação da acuidade visual e do campo visual após tratamento adequado, os pacientes que não apresentam este sinal devem apresentar recuperação completa ou muito acentuada das funções visuais. Entretanto, a observação clínica da $\mathrm{AB}$ do nervo óptico nas síndromes quiasmáticas é um dado semiológico subjetivo e qualitativo e sua importância como parâmetro de prognósti- co visual é mais fidedigna quando avaliada por um examinador experiente. Os métodos que avaliam a integridade anatômica ou funcional da via óptica são promissores para avaliação do prognóstico visual nas síndromes compressivas e sua importância é devido à possibilidade de recuperação pós-tratamento das funções visuais nas afecções compressivas da via óptica. Por outro lado, o padrão de perda na atrofia em banda pode representar um modelo para avaliar a capacidade de qualquer instrumento em medir a perda da CFNR, especialmente nas regiões nasal e temporal do DO.

Usando este modelo de perda neural, conduzimos um estudo prospectivo para avaliar desempenho diagnóstico do GDx em pacientes com hemianopsia temporal bem estabelecida e AB do nervo óptico secundária a compressão quiasmática, a fim de avaliar a capacidade deste instrumento para identificar o padrão típico de perda de fibras nervosas observadas nesses pacientes. Os valores da CFNR medidos pelo GDx nos olhos com atrofia em banda do nervo óptico foram significativamente diferentes das do grupo controle em todas as regiões, menos a temporal. O banco de dados presente no software aparelho, não conseguiu identificar a maioria das anormalidades nas regiões temporal e nasal em pacientes, apesar do fato de que estas áreas são claramente anormais nos olhos com atrofia do nervo óptico. Os resultados deste estudo foram importantes pois chamaram a atenção para o fato de que a avaliação da CFNR com a polarimetria a laser (GDx) deve ser interpretada com cautela no estudo de doenças oculares que levam à perda axonal predominantemente nas áreas nasal e temporal do disco óptico. 


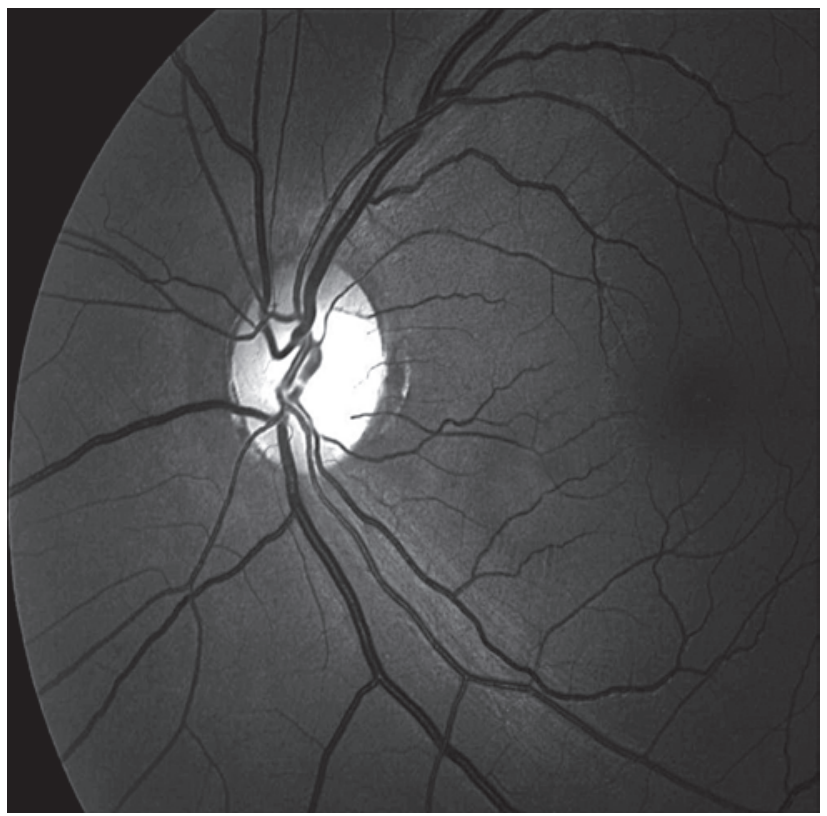

Figura 8: Fotografia do fundo de olho de paciente com hemianopsia temporal e atrofia em banda do nervo óptico. Observe a ausência de fibras nos setores nasal e temporal do disco e rarefação das fibras nas arcadas superior e inferior

A TCO por outro lado demonstra um desempenho superior, sendo capaz de identificar a perda da CFNR em todos os quadrantes, particularmente o quadrante temporal. Na tentativa de melhorar o desempenho diagnóstico do GDx, dois implementos no software foram realizados em duas novas versões recém-disponíveis com mecanismos compensadores da polarização corneana, o GDx "VCC" (compensador variável de córnea) e o GDx "ECC" (compensação aperfeiçoada da córnea). Com intuito de avaliar se estes mecanismos compensadores da polarização corneana resultaram numa melhora da habilidade diagnóstica do GDx, conduzimos dois estudos. $^{27,72}$ Os resultados demonstraram que o compensador variável de córnea melhorou a performance diagnóstica para detecção da perda da CFNR na espessura média e nos quadrantes superior, inferior, nasal. Entretanto, quando comparado ao Stratus-OCT, GDx "VCC" continuou tendo um pobre desempenho na detecção da perda axonal no setor temporal do disco óptico. Em um outro estudo, ${ }^{(27)}$ foi realizado uma comparação direta da habilidade diagnóstica em pacientes com atrofia em banda do nervo óptico destas duas novas versões de GDx, o "VCC" e o "ECC". Os resultados demonstraram que o implemento da compensação aperfeiçoada da córnea ("ECC") não promoveu uma melhora desempenho da perda da CFNR nos setores nasal e temporal neste grupo de pacientes. Portanto, a TCO parece ser uma tecnologia mais útil na detecção da perda axonal nas afecções da via óptica anterior, sendo capaz de produzir medidas quantitativas, reprodutíveis e com boa acurácia da CFNR, incluindo o setor temporal do disco óptico.

Entretanto, apesar destes resultados animadores, alguns aspectos devem ser ressaltados. $\mathrm{O}$ desempenho da TCO TD na quantificação da perda axonal pela avaliação da CFNR através das medidas da espessura média e nos quatro quadrantes (superior, inferior, nasal e temporal) ter demonstrado bom desempenho diagnóstico, a análise das medidas nos 12 segmentos de 30 graus deve ser vista com cuidado. Em outro estudo, avaliamos a habilidade diagnóstica do aparelho baseado em banco de dados normativos do aparelho em pacientes com $\mathrm{AB}$ do nervo óptico. ${ }^{(73)}$ Os resultados demonstraram que o parâmetro espessura média foi o com maior sensibilidade para detecção de anormalidades nos olhos com a AB, seguido pelos parâmetros dos quadrantes temporal e nasal. O que chamou atenção é que os parâmetros dos segmentos de 30 graus, os valores de sensibilidade foram relativamente baixos. Sendo que falhas em detectar a perda pode ocorrer nos segmentos de 30 graus, especialmente no meridiano de 3 horas (temporal), justamente o setor sabidamente afetado.

Apesar destas ressalvas deve ser enfatizado que a TCO mostra claramente o afilamento da CFN em todos os setores, principalmente os setores nasal e temporal do disco óptico. ${ }^{(72,74-76)}$ Estudos demonstraram que existe correlação entre a gravidade da perda visual e as medidas de perda axonal retiniana seja pela CFNR seja pela espessura macular. ${ }^{(75,77-78)}$ A quantificação da perda neural nas compressões quiasmáticas é também importante no sentido de tentar estimar a possibilidade de recuperação visual. Para isso, é de fundamental importância estudos de correlação estrutura-função, ou seja, verificar como se correlacionam os defeitos permanentes do campo visual com a perda da CFNR. Assim, nos pacientes que apresentam perda de campo sem comprometimento das fibras nervosas existe a possibilidade de recuperação visual importante, ao passo que aqueles com lesão grave das fibras nervosas não apresentam recuperação. ${ }^{(77,79-81)}$

\section{Lesões do trato óptico e corpo geniculado lateral}

Lesões do trato óptico, embora infrequentes, são de grande importância pelas suas implicações diagnósticas. Representam o primeiro local na região retroquiasmática onde se produz defeitos de campo hemianópicos. As etiologias são principalmente os tumores supraselares, como os craniofaringeomas e os adenomas pituitários, podendo também ser causados por processos desmielinizantes, lesões vasculares e trauma. Clinicamente as lesões do trato óptico se apresentam com acuidade visual normal em ambos os olhos, hemianopsia homônima contralateral à lesão e defeito pupilar aferente no olho da hemianopsia temporal. ${ }^{(82,83)}$ Hemianopsia pode ser completa ou parcial e quando incompleta, os defeitos de campo são usualmente incongruentes, embora lesões vasculares possam ocasionalmente produzir lesões congruentes. ${ }^{\left({ }^{84}\right)}$

Lesões completas do trato óptico com hemianopsia homônima produzem alterações distintas no fundo de olho. No olho contralateral à lesão, o olho com a hemianopsia 
temporal, o nervo apresenta alterações típicas da atrofia em banda do nervo, de forma análoga àquela descrita acima para as lesões quiasmáticas. ${ }^{(8,7,8,82,85)}$ As alterações no olho ipsilateral, no entanto, são mais difíceis de detectar. O olho da hemianopsia nasal apresenta perda das fibras da retina temporal que penetram o disco óptico pelas arcadas superior e inferior. Estas fibras estão imbrincadas com outras da retina nasal que também trafegam as arcadas superior e inferior.A atrofia das fibras da hemirretina temporal não produz, portanto, a perda completa das fibras nas arcadas e apenas afilamento das mesmas. A alteração da CFRN das lesões do trato óptico é indistinguível daquela por lesão no corpo geniculado lateral.

Recentemente pudemos estudar dois pacientes com lesões do trato óptico estudados com auxílio do OCT. Demonstramos a perda predominante nos setores nasal e temporal do disco óptico no olho da hemianopsia temporal com preservação relativa dos quadrantes superior e inferior. ${ }^{(86)}$ No olho da hemianopsia nasal evidenciou-se a perda de fibras nos quadrantes superior e inferior do disco óptico, acometendo, portanto, as fibras da retina temporal que penetram o olho pelas arcadas superior e inferior. Os casos serviram para confirmar o valor da TCO na identificação da perda neural nestes pacientes. Embora os achados clínicos de palidez de papila e defeito aferente relativo possam auxiliar a separar os defeitos homônimos decorrentes de lesões do trato óptico ou do corpo geniculado lateral daqueles das radiações e do lobos occipitais, o OCT pode ser útil para definir a perda da CFNR em casos onde a observação da palidez é difícil.

Lesões que acometem o corpo geniculado lateral (CGL) são as mais raras entre as afecções da via óptica. A suspeita dessas lesões baseia-se no tipo de defeito campimétrico apresentado, associado ao padrão de perda da CFNR observado à oftalmoscopia, aos achados no exame das pupilas e aos achados neurológicos associados. O estudo de imagem pela tomografia computadorizada ou pela ressonância magnética tem a importante função de localizar a lesão e confirmar o diagnóstico. Entretanto, quando os achados aos exames de imagem são discretos, tais como ocorre em pequenas lesões isquêmicas do CGL, o diagnóstico pode ser difícil e depender fundamentalmente dos achados clínicos e do defeito campimétrico.

Em um estudo prévio demonstramos a utilidade da TCO na identificação da perda neural em paciente com lesão discreta no CGL, de difícil diagnóstico até pela imagem por ressonância magnética. A dificuldade era ainda maior pois o paciente tinha apenas um olho (outro olho em atrofia) o que dificultava a caracterização do tipo de hemianopsia. ${ }^{(87)}$ Após a identificação da perda da CFNR pelo OCT o exame de imagem por ressonância magnética foi repetido utilizando-se parâmetros técnicos especiais que facilitaram a identificação do CGL. O resultado desse novo exame confirmou nossa suspeita de lesão isquêmica do CGL à es- querda e serviu para esclarecer definitivamente a causa do defeito campimétrico. Embora a suspeita clínica tenha sido o fator decisivo para o diagnóstico, a informação obtida através da tomografia por coerência óptica revelou-se de importância fundamental dando suporte aos achados clínicos e reforçando a suspeita diagnóstica.

\section{Comentários conclusivos}

Avanços na semiologia tornaram cada vez mais possível a identificação e quantificação da perda neural retiniana pela análise cuidadosa da CFNR. Como discutido tal aspecto da semiologia assume fundamental importância não apenas em glaucoma como em diversas afecções da via óptica anterior. Avanços nas tecnologias disponíveis por um lado permitem uma maior acurácia diagnóstica mas também obrigam a constante atualização para se compreender as reais possibilidades e limitações de cada uma delas. O oftalmologista deve estar atento para tais métodos semiológicos, suas possibilidades e causas de interpretações errôneas para tirar o melhor proveito dos mesmos no auxílio diagnóstico e condução clínica adequada aos seus pacientes com afecções da via óptica anterior.

\section{ReFERÊNCIAS}

1. Hoyt WF, Newman NM. The earliest observable defect in glaucoma? Lancet. 1972;1(7752):692-3.

2. Hoyt WF, Frisén L, Newman NM. Fundoscopy of nerve fiber layer defects in glaucoma. Invest Ophthalmol. $1973 ; 12(11): 814-29$

3. Drance SM, Airaksinen PJ, Price M, Schulzer M, Douglas GR, Tansley BW. The correlation of functional and structural measurements in glaucoma patients and normal subjects. Am J Ophthalmol. 1986;102(5):612-6.

4. Sommer A, Katz J, Quigley HA, Miller NR, Robin AL, Richter RC, Witt KA. Clinically detectable nerve fiber atrophy precedes the onset of glaucomatous field loss. Arch Ophthalmol. 1991;109(1):77-83.

5. Sommer A, Quigley HA, Robin AL, Miller NR, Katz J, Arkell S. Evaluation of nerve fiber layer assessment. Arch Ophthalmol. 1984;102(12):1766-71.

6. Frisén L, Hoyt WF. Insidious atrophy of retinal nerve fibers in multiple sclerosis. Funduscopic identification in patients with and without visual complaints. Arch Ophthalmol. 1974;92(2):91-7.

7. Leal BC, Moura FC, Monteiro ML. Comparacão entre o polarímetro de varredura a laser, a tomografia de coerência óptica 1 e o Stratus-OCT na detecção da perda axonal da atrofia em banda do nervo óptico. Arq Bras Oftalmol. 2006;69(4):531-7.

8. Monteiro ML, Medeiros FA, Ostroscki MR. Quantitative analysis of axonal loss in band atrophy of the optic nerve using scanning laser polarimetry. $\mathrm{Br} \mathrm{J}$ Ophthalmol. 2003;87(1):32-7.

9. Chan CK, Cheng AC, Leung CK, Cheung CY, Yung AY, Gong B, Lam DS. Quantitative assessment of optic nerve head morphology and retinal nerve fibre layer in non-arteritic anterior ischaemic optic neuropathy with optical coherence tomography and confocal scanning laser ophthalmoloscopy. Br J Ophthalmol. 2009;93(6):731-5. Comment in: Br J Ophthalmol. 2009;93(6):703.

10. MacFadyen DJ, Drance SM, Douglas GR, Airaksinen PJ, Mawson DK, Paty DW. The retinal nerve fiber layer, neuroretinal rim area, and visual evoked potentials in MS. Neurology. 1988;38(9):1353-8. 
11. Trip SA, Schlottmann PG, Jones SJ, Altmann DR, GarwayHeath DF, Thompson AJ, et al. Retinal nerve fiber layer axonal loss and visual dysfunction in optic neuritis. Ann Neurol. 2005;58(3):383-91.

12. Walsh FB, Hoyt WF, Miller NR, editors. Walsh and Hoyt's Clinical neuro-ophthalmology. 6th ed. Baltimore: Williams \& Wilkins; 1982. p.26-30.

13. Varma R, Skaf M, Barron E. Retinal nerve fiber layer thickness in normal human eyes. Ophthalmology. 1996;103(12):21149. Erratum in: Ophthalmology 1997;104(2):174.

14. Hoyt WF, Schlicke B, Eckelhoff RJ. Fundoscopic appearance of a nerve-fibre-bundle defect. Br J Ophthalmol. 1972;56(8):577-83.

15. Jonas JB, Nguyen NX, Naumann GO. The retinal nerve fiber layer in normal eyes. Ophthalmology. 1989;96(5):627-32.

16. Quigley HA, Addicks EM. Quantitative studies of retinal nerve fiber layer defects. Arch Ophthalmol. 1982;100(5):807-14.

17. Weinreb RN, Shakiba S, Zangwill L. Scanning laser polarimetry to measure the nerve fiber layer of normal and glaucomatous eyes. Am J Ophthalmol. 1995;119(5):627-36.

18. Zhou Q, Knighton RW. Light scattering and form birefringence of parallel cylindrical arrays that represent cellular organelles of the retinal nerve fiber layer. Appl Opt. 1997;36(10):2273-85.

19. Zangwill L, Berry CA, Garden VS, Weinreb RN. Reproducibility of retardation measurements with the nerve fiber analyzer II. J Glaucoma. 1997;6(6):384-9.

20. Hoh ST, Ishikawa H, Greenfield DS, Liebmann JM, Chew SJ, Ritch R. Peripapillary nerve fiber layer thickness measurement reproducibility using scanning laser polarimetry. J Glaucoma. 1998;7(1):12-5.

21. Steel DH, Waldock A. Measurement of the retinal nerve fibre layer with scanning laser polarimetry in patients with previous demyelinating optic neuritis. J Neurol Neurosurg Psychiatry. 1998;64(4):505-9.

22. Medeiros FA, Susanna R Jr. Retinal nerve fiber layer loss after traumatic optic neuropathy detected by scanning laser polarimetry. Arch Ophthalmol. 2001;119(6):920-1.

23. Colen TP, van Everdingen JA, Lemij HG. Axonal loss in a patient with anterior ischemic optic neuropathy as measured with scanning laser polarimetry. Am J Ophthalmol. 2000;130(6):847-50.

24. Weinreb RN, Shakiba S, Sample PA, Shahrokni S, van Horn S, Garden VS, et al. Association between quantitative nerve fiber layer measurement and visual field loss in glaucoma. Am J Ophthalmol. 1995;120(6):732-8.

25. Kogure S, Iijima H, Tsukahara S. A new parameter for assessing the thickness of the retinal nerve fiber layer for glaucoma diagnosis. Eur J Ophthalmol. 1999;9(2):93-8.

26. Kogure S, Chiba T, Kinoshita T, Kowa H, Tsukahara S. Effects of artefacts on scanning laser polarimetry of retinal nerve fibre layer thickness measurement. Br J Ophthalmol. 2000;84(9):1013-7.

27. Monteiro ML, Moura FC, Medeiros FA. Scanning laser polarimetry with enhanced corneal compensation for detection of axonal loss in band atrophy of the optic nerve. Am J Ophthalmol. 2008;145(4):747-54.

28. Huang D, Swanson EA, Lin CP, Schuman JS, Stinson WG, Chang W, et al. Optical coherence tomography. Science. 1991;254(5035):1178-81.

29. Stratus OCT Model 3000 User Manual. Dublin, CA: Carl Zeiss Meditec Inc; 2003.

30. Blumenthal EZ, Williams JM, Weinreb RN, Girkin CA, Berry CC, Zangwill LM. Reproducibility of nerve fiber layer thickness measurements by use of optical coherence tomography. Ophthalmology. 2000;107(12):2278-82.

31. Schuman JS, Pedut-Kloizman T, Hertzmark E, Hee MR, Wilkins JR, Coker JG, et al. Reproducibility of nerve fiber layer thickness measurements using optical coherence tomography. Ophthalmology. 1996;103(11):1889-98. Comment in: Ophthalmology. 1997;104(10):1530-1.

32. Costa-Cunha LV, Cunha LP, Malta RF, Monteiro ML. Comparison of Fourier-domain and time-domain optical coherence tomography in the detection of band atrophy of the optic nerve. Am J Ophthalmol. 2009;147(1):56-63.e2.
33. Wojtkowski M, Bajraszewski T, Gorczynska I, Targowski P, Kowalczyk A, Wasilewski W, Radzewicz C. Ophthalmic imaging by spectral optical coherence tomography. Am J Ophthalmol. 2004;138(3):412-9.

34. Wojtkowski M, Srinivasan V, Ko T, Fujimoto J, Kowalczyk A, Duker J. Ultrahigh-resolution, high-speed, Fourier domain optical coherence tomography and methods for dispersion compensation. Opt Express. 2004;12(11):2404-22.

35. Christopoulos V, Kagemann L, Wollstein G, Ishikawa H, Gabriele ML, Wojtkowski M, et al. In vivo corneal high-speed, ultra high-resolution optical coherence tomography. Arch Ophthalmol. 2007;125(8):1027-35.

36. Gabriele ML, Ishikawa H, Wollstein G, Bilonick RA, Kagemann L, Wojtkowski M, et al. Peripapillary nerve fiber layer thickness profile determined with high speed, ultrahigh resolution optical coherence tomography high-density scanning. Invest Ophthalmol Vis Sci. 2007;48(7):3154-60.

37. Anderson DR. Ascending and descending optic atrophy produced experimentally in squirrel monkeys. Am J Ophthalmol. 1973;76(5):693-711.

38. Miller NR, Newman NJ. Walsh a\& Hoyt's clinical neuro-ophthalmology. 6th ed. Philadelphia: Lippincott Williams \& Wilkins; c2005. V. 1. p. 209-11.

39. Siger M, Dziegielewski K, Jasek L, Bieniek M, Nicpan A, Nawrocki J, Selmaj K. Optical coherence tomography in multiple sclerosis: thickness of the retinal nerve fiber layer as a potential measure of axonal loss and brain atrophy. J Neurol. 2008;255(10):1555-60.

40. Costello F, Coupland S, Hodge W, Lorello GR, Koroluk J, Pan YI, et al. Quantifying axonal loss after optic neuritis with optical coherence tomography. Ann Neurol. 2006;59(6):963-9.

41. Fisher JB, Jacobs DA, Markowitz CE, Galetta SL, Volpe NJ, Nano-Schiavi ML, et al. Relation of visual function to retinal nerve fiber layer thickness in multiple sclerosis. Ophthalmology. 2006;113(2):324-32. Comment in: Ophthalmology. 2007;114(1):200.

42. Petzold A, de Boer JF, Schippling S, Vermersch P, Kardon R, Green A, et al. Optical coherence tomography in multiple sclerosis: a systematic review and meta-analysis. Lancet Neurol. 2010;9(9):921-32. Erratum in: Lancet Neurol. 2010;9(11):1045.

43. Trip SA, Schlottmann PG, Jones SJ, Kallis C, Altmann DR, Garway-Heath DF, et al. Scanning laser polarimetry quantification of retinal nerve fiber layer thinning following optic neuritis. J Neuroophthalmol. 2010;30(3):235-42.

44. Zaveri MS, Conger A, Salter A, Frohman TC, Galetta SL, Markowitz CE, et al. Retinal imaging by laser polarimetry and optical coherence tomography evidence of axonal degeneration in multiple sclerosis. Arch Neurol. 2008;65(7):924-8.

45. Wingerchuk DM, Hogancamp WF, O'Brien PC, Weinshenker BG. The clinical course of neuromyelitis optica (Devic's syndrome). Neurology. 1999;53(5):1107-14.

46. Naismith RT, Tutlam NT, Xu J, Klawiter EC, Shepherd J, Trinkaus K, et al. Optical coherence tomography differs in neuromyelitis optica compared with multiple sclerosis. Neurology. 2009;72(12):1077-82.

47. Monteiro ML. Anterior ischemic optic neuropathy: a comparison of the optic disc area of patients with the arteritic and non-arteritic forms of the disease and that of normal controls. Arq Bras Oftalmol. 2006;69(6):805-10.

48. Bellusci C, Savini G, Carbonelli M, Carelli V, Sadun AA, Barboni P. Retinal nerve fiber layer thickness in nonarteritic anterior ischemic optic neuropathy: OCT characterization of the acute and resolving phases. Graefes Arch Clin Exp Ophthalmol. 2008;246(5):641-7.

49. Contreras I, Rebolleda G, Noval S, Muñoz-Negrete FJ. Optic disc evaluation by optical coherence tomography in nonarteritic anterior ischemic optic neuropathy. Invest Ophthalmol Vis Sci. 2007;48(9):4087-92.

50. Nagai-Kusuhara A, Nakamura M, Kanamori A, Nakanishi Y, Kusuhara S, Negi A. Evaluation of optic nerve head configuration in various types of optic neuropathy with Heidelberg Retina Tomograph. Eye (Lond). 2008;22(9):1154-60. 
51. Deleón-Ortega J, Carroll KE, Arthur SN, Girkin CA. Correlations between retinal nerve fiber layer and visual field in eyes with nonarteritic anterior ischemic optic neuropathy. Am J Ophthalmol. 2007;143(2):288-94.

52. Danesh-Meyer HV, Carroll SC, Ku JY, Hsiang J, Gaskin B, Gamble GG, Savino PJ. Correlation of retinal nerve fiber layer measured by scanning laser polarimeter to visual field in ischemic optic neuropathy. Arch Ophthalmol. 2006;124(12):1720-6.

53. Imes RK, Schatz H, Hoyt WF, Monteiro ML, Narahara M. Evolution of optociliary veins in optic nerve sheath meningioma. Evolution. Arch Ophthalmol. 1985;103(1):59-60.

54. Monteiro ML, Goncalves AC, Silva CT, Moura JP, Ribeiro CS, Gebrim EM. Diagnostic ability of Barrett's index to detect dysthyroid optic neuropathy using multidetector computed tomography. Clinics (Sao Paulo). 2008;63(3):301-6.

55. Mayo GL, Carter JE, McKinnon SJ. Bilateral optic disk edema and blindness as initial presentation of acute lymphocytic leukemia. Am J Ophthalmol. 2002;134(1):141-2.

56. Coppeto JR, Monteiro ML, Cannarozzi DB. Optic neuropathy associated with chronic lymphomatous meningitis. J Clin Neuroophthalmol. 1988:8(1):39-45.

57. Votruba M, Thiselton D, Bhattacharya SS. Optic disc morphology of patients with OPA1 autosomal dominant optic atrophy. Br J Ophthalmol. 2003;87(1):48-53.

58. Kim TW, Hwang JM. Stratus OCT in dominant optic atrophy: features differentiating it from glaucoma. J Glaucoma. 2007;16(8):655-8.

59. Newman NJ, Lott MT, Wallace DC. The clinical characteristics of pedigrees of Leber's hereditary optic neuropathy with the 11778 mutation. Am J Ophthalmol. 1991;111(6):750-62.

60. Mackey DA, Buttery RG. Leber hereditary optic neuropathy in Australia. Aust N Z J Ophthalmol. 1992;20(3):177-84.

61. Barboni P, Savini G, Valentino ML, Montagna P, Cortelli P, De Negri AM, et al. Retinal nerve fiber layer evaluation by optical coherence tomography in Leber's hereditary optic neuropathy. Ophthalmology. 2005;112(1):120-6.

62. Walsh FB, Hoyt WF, Miller NR, editors. Walsh and Hoyt's Clinical neuro-ophthalmology. 6 th ed. Baltimore: Williams \& Wilkins; 1982. p. 254-60.

63. Orssaud C, Roche O, Dufier JL. Nutritional optic neuropathies. J Neurol Sci. 2007;262(1-2):158-64. Review.

64. Kee C, Hwang JM. Optical coherence tomography in a patient with tobacco-alcohol amblyopia. Eye (Lond). 2008;22(3):469-70.

65. Bhatnagar A, Sullivan C. Tobacco-alcohol amblyopia: can OCT predict the visual prognosis? Eye (Lond). 2009;23(7):1616-8.

66. Moura FC, Monteiro ML. Evaluation of retinal nerve fiber layer thickness measurements using optical coherence tomography in patients with tobacco-alcohol-induced toxic optic neuropathy. Indian J Ophthalmol. 2010;58(2):143-6.

67. Vessani RM, Cunha LP, Monteiro ML. Progressive macular thinning after indirect traumatic optic neuropathy documented by optical coherence tomography. Br J Ophthalmol. 2007;91(5):697-8.

68. Cunha LP, Costa-Cunha LV, Malta RF, Monteiro ML. Comparison between retinal nerve fiber layer and macular thickness measured with OCT detecting progressive axonal loss following traumatic optic neuropathy. Arq Bras Oftalmol. 2009;72(5):622-5.

69. Monteiro ML, Zambon BK, Cunha LP. Predictive factors for the development of visual loss in patients with pituitary macroadenomas and for visual recovery after optic pathway decompression. Can J Ophthalmol. 2010;45(4):404-8.

70. Unsöld R, Hoyt WF. Band atrophy of the optic nerve. The histology of temporal hemianopsia. Arch Ophthalmol. 1980;98(9):1637-8.

71. Mikelberg FS, Yidegiligne HM. Axonal loss in band atrophy of the optic nerve in craniopharyngioma: a quantitative analysis. Can J Ophthalmol. 1993;28(2):69-71.

72. Monteiro ML, Moura FC. Comparison of the GDx VCC scanning laser polarimeter and the stratus optical coherence tomograph in the detection of band atrophy of the optic nerve. Eye (Lond). 2008;22(5):641-8.
73. Monteiro ML, Moura FC, Medeiros FA. Diagnostic ability of optical coherence tomography with a normative database to detect band atrophy of the optic nerve. Am J Ophthalmol. 2007;143(5):896-9.

74. Kanamori A, Nakamura M, Matsui N, Nagai A, Nakanishi Y, Kusuhara S, et al. Optical coherence tomography detects characteristic retinal nerve fiber layer thickness corresponding to band atrophy of the optic discs. Ophthalmology. 2004;111(12):2278-83. Comment in: Ophthalmology. 2005;112(11):2055-6; author reply 2056-7.

75. Danesh-Meyer HV, Carroll SC, Foroozan R, Savino PJ, Fan J, Jiang Y, Vander Hoorn S. Relationship between retinal nerve fiber layer and visual field sensitivity as measured by optical coherence tomography in chiasmal compression. Invest Ophthalmol Vis Sci. 2006;47(11):4827-35.

76. Moura FC, Medeiros FA, Monteiro ML. Evaluation of macular thickness measurements for detection of band atrophy of the optic nerve using optical coherence tomography. Ophthalmology. 2007;114(1):175-81.

77. Monteiro ML, Costa-Cunha LV, Cunha LP, Malta RF. Correlation between macular and retinal nerve fibre layer Fourierdomain OCT measurements and visual field loss in chiasmal compression. Eye (Lond). 2010;24(8):1382-90.

78. Moura FC, Costa-Cunha LV, Malta RF, Monteiro ML. Relationship between visual field sensitivity loss and quadrantic macular thickness measured with Stratus-Optical coherence tomography in patients with chiasmal syndrome. Arq Bras Oftalmol. 2010;73(5):409-13.

79. Danesh-Meyer HV, Papchenko T, Savino PJ, Law A, Evans J, Gamble GD. In vivo retinal nerve fiber layer thickness measured by optical coherence tomography predicts visual recovery after surgery for parachiasmal tumors. Invest Ophthalmol Vis Sci. 2008;49(5):1879-85.

80. Monteiro ML, Cunha LP, Costa-Cunha LV, Maia OO Jr, Oyamada MK. Relationship between optical coherence tomography, pattern electroretinogram and automated perimetry in eyes with temporal hemianopia from chiasmal compression. Invest Ophthalmol Vis Sci. 2009;50(8):3535-41.

81. Jacob M, Raverot G, Jouanneau E, Borson-Chazot F, Perrin $\mathrm{G}$, Rabilloud M, et al. Predicting visual outcome after treatment of pituitary adenomas with optical coherence tomography. Am J Ophthalmol. 2009;147(1):64-70.e2. Comment in: Am J Ophthalmol. 2009;147(6):1103-4; author reply 1104.

82. Newman SA, Miller NR. Optic tract syndrome. Neuro-ophthalmologic considerations. Arch Ophthalmol. 1983;101(8):1241-50.

83. Kardon R, Kawasaki A, Miller NR. Origin of the relative afferent pupillary defect in optic tract lesions. Ophthalmology. 2006;113(8):1345-53.

84. Kedar S, Zhang X, Lynn MJ, Newman NJ, Biousse V. Congruency in homonymous hemianopia. Am J Ophthalmol. 2007;143(5):772-80. Comment in: Am J Ophthalmol. 2007;143(5):856-8. Am J Ophthalmol. 2007;144(5):786.

85. Monteiro ML, Leal BC, Moura FC, Vessani RM, Medeiros FA. Comparison of retinal nerve fibre layer measurements using optical coherence tomography versions 1 and 3 in eyes with band atrophy of the optic nerve and normal controls. Eye (Lond). 2007;21(1):16-22.

86. Monteiro ML, Hokazone K. Retinal nerve fiber layer loss documented by optical coherence tomography in patients with optic tract lesions. Rev Bras Oftalmol. 2009;68(1):48-52.

87. Moura FC, Lunardelli P, Leite CC, Monteiro ML. Hemianopsia por lesão no corpo geniculado lateral. Importância diagnóstica da análise da camada de fibras nervosas pela tomografia por coerência óptica: relato de caso. Arq Bras Oftalmol. 2005;68(6):860-3.

Endereço para correspondência

Mário L. R. Monteiro

Av. Angélica,n ${ }^{\circ} 1757$ - conj. 61

CEP 01227-200 - São Paulo (SP), Brasil 